\title{
A Fixed Point Approach to the Stability of a General Mixed AQCQ-Functional Equation in Non-Archimedean Normed Spaces
}

\author{
Tian Zhou Xu, ${ }^{1}$ John Michael Rassias, ${ }^{2}$ and Wan Xin $X \mathbf{u}^{3}$ \\ ${ }^{1}$ Department of Mathematics, School of Science, Beijing Institute of Technology, Beijing 100081, China \\ ${ }^{2}$ Sections of Mathematics and Informatics, Pedagogical Department E.E., National and Capodistrian \\ University of Athens, 4, Agamemnonos Str., Aghia Paraskevi, 15342 Athens, Greece \\ ${ }^{3}$ School of Communication and Information Engineering, University of Electronic Science and Technology \\ of China, Chengdu 611731, China
}

Correspondence should be addressed to Tian Zhou Xu, xutianzhou@bit.edu.cn

Received 28 June 2010; Accepted 2 September 2010

Academic Editor: Xue Zhong He

Copyright (C 2010 Tian Zhou Xu et al. This is an open access article distributed under the Creative Commons Attribution License, which permits unrestricted use, distribution, and reproduction in any medium, provided the original work is properly cited.

Using the fixed point methods, we prove the generalized Hyers-Ulam stability of the general mixed additive-quadratic-cubic-quartic functional equation $f(x+k y)+f(x-k y)=k^{2} f(x+y)+k^{2} f(x-$ $y)+2\left(1-k^{2}\right) f(x)+\left(\left(k^{4}-k^{2}\right) / 12\right)[f(2 y)+f(-2 y)-4 f(y)-4 f(-y)]$ for a fixed integer $k$ with $k \neq 0, \pm 1$ in non-Archimedean normed spaces.

\section{Introduction}

A basic question in the theory of functional equations is as follows: when is it true that a function, which approximately satisfies a functional equation, must be close to an exact solution of the equation?

If the problem accepts a unique solution, we say the equation is stable. The first stability problem concerning group homomorphisms was raised by Ulam [1] in 1940 and affirmatively solved by Hyers [2]. The result of Hyers was generalized by Aoki [3] for approximate additive mappings and by Th. M. Rassias [4] for approximate linear mappings by allowing the Cauchy difference operator $\operatorname{CD} f(x, y)=f(x+y)-[f(x)+f(y)]$ to be controlled by $\epsilon\left(\|x\|^{p}+\|y\|^{p}\right)$. In 1994, a generalization of Rassias' theorem was obtained by Găvruţa [5], who replaced $\epsilon\left(\|x\|^{p}+\|y\|^{p}\right)$ by a general control function $\varphi(x, y)$. In addition, J. M. Rassias et al. [6-14] generalized the Hyers stability result by introducing two weaker conditions controlled by a product of different powers of norms and a mixed productsum of powers of norms, respectively. Recently, several further interesting discussions, 
modifications, extensions, and generalizations of the original problem of Ulam have been proposed (see, e.g., [11-13,15-28] and the references therein).

In 1897, Hensel discovered the $p$-adic numbers as a number theoretical analogue of power series in complex analysis. The most important examples of non-Archimedean spaces are $p$-adic numbers. A key property of $p$-adic numbers is that they do not satisfy the Archimedean axiom: for all $x, y>0$, there exists an integer $n$ such that $x<n y$. It turned out that non-Archimedean spaces have many nice applications $[18,29,30]$.

During the last three decades, the theory of non-Archimedean spaces has gained the interest of physicists for their research particularly in problems coming from quantum physics, $p$-adic strings and superstrings [18]. Although many results in the classical normed space theory have a non-Archimedean counterpart, their proofs are essentially different and require an entirely new kind of intuition. One may note that for $|n| \leq 1$ in each valuation field, every triangle is isosceles and there may be no unit vector in a non-Archimedean normed space [18]. These facts show that the non-Archimedean framework is of special interest.

In 1996, Isac and Th. M. Rassias [31] were the first to provide applications of stability theory of functional equations for the proof of new fixed point theorems with applications. The stability problems of several various functional equations have been extensively investigated by a number of authors using fixed point methods (see [12, 19, 26, 32]).

The functional equation

$$
f(x+y)+f(x-y)=2 f(x)+2 f(y)
$$

is said to be a quadratic functional equation because the quadratic function $f(x)=x^{2}$ is a solution of the functional equation (1.1). Every solution of the quadratic functional equation is said to be a quadratic mapping. A quadratic functional equation was used to characterize inner product spaces.

In 2001, J. M. Rassias [6] introduced the cubic functional equation

$$
f(x+2 y)-3 f(x+y)+3 f(x)-f(x-y)=6 f(y)
$$

and established the solution of the Ulam stability problem for these cubic mappings. It is easy to show that the function $f(x)=x^{3}$ satisfies the functional equation (1.2) which is called a cubic functional equation, and every solution of the cubic functional equation is said to be a cubic mapping. The quartic functional equation

$$
f(x+2 y)+f(x-2 y)=4 f(x+y)+4 f(x-y)+6 f(x)+24 f(y)
$$

was introduced by J. M. Rassias [8]. It is easy to show that the function $f(x)=x^{4}$ is the solution of (1.3). Every solution of the quartic functional equation is said to be a quartic mapping. Park [26] proved the generalized Hyers-Ulam stability of the following additivequadratic-cubic-quartic functional equation (briefly, AQCQ-functional equation):

$$
f(x+2 y)+f(x-2 y)=4 f(x+y)+4 f(x-y)-6 f(x)+f(2 y)+f(-2 y)-4 f(y)-4 f(-y)
$$

in non-Archimedean normed spaces. 
In [10], Ravi et al. introduced a general mixed-type AQCQ-functional equation

$$
\begin{aligned}
f(x+k y)+f(x-k y)= & k^{2} f(x+y)+k^{2} f(x-y)+2\left(1-k^{2}\right) f(x) \\
& +\frac{k^{4}-k^{2}}{12}[f(2 y)+f(-2 y)-4 f(y)-4 f(-y)],
\end{aligned}
$$

which is a generalized form of the AQCQ-functional equation (1.4) and obtained its general solution and generalized Hyers-Ulam stability for a fixed integer $k$ with $k \neq 0, \pm 1$ in Banach spaces.

In this paper, using the fixed point method, we prove the generalized Hyers-Ulam stability of the general mixed AQCQ-functional equation (1.5) in non-Archimedean normed spaces.

\section{Preliminaries}

We recall some basic facts concerning non-Archimedean space and some preliminary results (see $[15,20-22,26])$.

A valuation is a function $|\cdot|$ from a field $\mathbb{K}$ into $[0, \infty)$ such that 0 is the unique element having the 0 valuation, $|r s|=|r| \cdot|s|$, and the triangle inequality holds, that is,

$$
|r+s| \leq|r|+|s|, \quad \forall r, s \in \mathbb{K}
$$

A field $\mathbb{K}$ is called a valued field if $\mathbb{K}$ carries a valuation. The usual absolute values of $\mathbb{R}$ and $\mathbb{C}$ are examples of valuations.

Let us consider a valuation which satisfies a stronger condition than the triangle inequality.

Definition 2.1. Let $\mathbb{K}$ be a field. A non-Archimedean valuation on $\mathbb{K}$ is a function $|\cdot|: \mathbb{K} \rightarrow$ $[0, \infty)$ such that for any $r, s \in \mathbb{K}$, one has the following:

(i) $|r| \geq 0$, and equality holds if and only if $r=0$;

(ii) $|r s|=|r| \cdot|s|$;

(iii) $|r+s| \leq \max \{|r|,|s|\}$.

The condition (iii) is called the strong triangle inequality. Clearly, $|1|=|-1|=1$ and $|n| \leq 1$ for all $n \in \mathbb{N}$. A trivial example of a non-Archimedean valuation is the function $|\cdot|$ taking everything except for 0 into 1 and $|0|=0$.

Throughout this paper, we assume that the base field is a non-Archimedean field and hence call it simply a field.

Definition 2.2 (see $[15,22])$. Let $X$ be a linear space over a field $\mathbb{K}$ with a non-Archimedean valuation $|\cdot|$. A function $\|\cdot\|: X \rightarrow[0, \infty)$ is a non-Archimedean norm if it satisfies the following conditions:

(i) $\|x\|=0$ if and only if $x=0$,

(ii) $\|r x\|=|r|\|x\|$ for all $r \in \mathbb{K}$ and $x \in X$, 
(iii) the strong triangle inequality;

$$
\|x+y\| \leq \max \{\|x\|,\|y\|\}, \quad \forall x, y \in X
$$

Then $(X,\|\cdot\|)$ is called a non-Archimedean normed space.

Definition 2.3. Let $X$ be a non-Archimedean normed space. Let $\left\{x_{n}\right\}$ be a sequence in $X$. Then, $\left\{x_{n}\right\}$ is said to be convergent if there exists $x \in X$ such that $\lim _{n \rightarrow \infty}\left\|x_{n}-x\right\|=0$. In that case, $x$ is called the limit of the sequence $\left\{x_{n}\right\}$, and one denotes it by $\lim _{n \rightarrow \infty} x_{n}=x$.

A sequence $\left\{x_{n}\right\}$ in $X$ is said to be a Cauchy sequence if $\lim _{n \rightarrow \infty}\left\|x_{n+p}-x_{n}\right\|=0$ for all $p=1,2,3, \ldots$. Due to the fact that

$$
\left\|x_{n}-x_{m}\right\| \leq \max \left\{\left\|x_{j+1}-x_{j}\right\|: m \leq j \leq n-1\right\} \quad(n>m),
$$

a sequence $\left\{x_{n}\right\}$ is Cauchy if and only if $\left\{x_{n+1}-x_{n}\right\}$ converges to zero in a non-Archimedean normed space.

It is known that every convergent sequence in a non-Archimedean normed space is a Cauchy sequence. If every Cauchy sequence in $X$ converges, then the non-Archimedean normed space $X$ is called a non-Archimedean Banach space.

Let $X$ be a set. A function $d: X \times X \rightarrow[0, \infty]$ is called a generalized metric on $X$ if $d$ satisfies the following:

(1) $d(x, y)=0$ if and only if $x=y$;

(2) $d(x, y)=d(y, x)$ for all $x, y \in X$;

(3) $d(x, y) \leq d(x, z)+d(y, z)$ for all $x, y, z \in X$.

For explicit later use, we recall a fundamental result in fixed point theory.

Theorem 2.4 (The fixed point alternative theorem, see $[12,26,33])$. Let $(\Omega, d)$ be a complete generalized metric space, and let, $J: \Omega \rightarrow \Omega$ be a strictly contractive mapping with Lipschitz constant $0<L<1$, that is,

$$
d(J x, J y) \leq L d(x, y) \quad \forall x \in X
$$

Then, for each given $x \in \Omega$, either

$$
d\left(J^{n} x, J^{n+1} x\right)=\infty \quad \forall n \geq 0
$$

or

$$
d\left(J^{n} x, J^{n+1} x\right)<\infty \quad \forall n \geq n_{0}
$$

for some nonnegative integer $n_{0}$. Actually, if the second alternative holds, then the sequence $\left\{J^{n} x\right\}$ converges to a fixed point $y^{*}$ of $J$, and

(1) $y^{*}$ is the unique fixed point of $J$ in the set $\Delta=\left\{y \in \Omega: d\left(J^{n_{0}} x, y\right)<\infty\right\}$;

(2) $d\left(y, y^{*}\right) \leq(1 /(1-L)) d(y, J y)$ for all $y \in \Delta$. 


\section{Generalized Hyers-Ulam Stability of the Functional Equation}

From now on, unless otherwise stated, we will assume that $X$ is a non-Archimedean normed space and $Y$ is a non-Archimedean Banach space. Utilizing the fixed point alternative, we will establish generalized Hyers-Ulam stability for the generalized mixed AQCQ-functional equation in non-Archimedean Banach space. For convenience, we use the following abbreviation for a given function $f: X \rightarrow Y$ :

$$
\begin{aligned}
D f(x, y):= & f(x+k y)+f(x-k y)-k^{2} f(x+y)-k^{2} f(x-y)-2\left(1-k^{2}\right) f(x) \\
& -\frac{k^{4}-k^{2}}{12}[f(2 y)+f(-2 y)-4 f(y)-4 f(-y)]
\end{aligned}
$$

for all $x, y \in X$.

Theorem 3.1. Let $\varphi: X \times X \rightarrow[0, \infty)$ be a function such that there exists an $L<1$ with

$$
\varphi(x, y) \leq \frac{L}{|2|} \varphi(2 x, 2 y)
$$

for all $x, y \in X$. Let $f: X \rightarrow Y$ be an odd function satisfying

$$
\|D f(x, y)\| \leq \varphi(x, y)
$$

for all $x, y \in X$. Then there exists a unique additive function $A: X \rightarrow Y$ such that

$$
\|f(2 x)-8 f(x)-A(x)\| \leq \frac{L}{|2|-|2| L} \tilde{\varphi}(x)
$$

for all $x \in X$, where

$$
\begin{array}{r}
\tilde{\varphi}(x):=\frac{1}{\left|k^{4}-k^{2}\right|} \max \left\{\left|2 k^{2}\right| \varphi(2 x, x),|2| \varphi(x, 2 x),|2| \varphi((1+k) x, x),|2| \varphi((1-k) x, x),\right. \\
\left.\left|k^{2}\right| \varphi(2 x, 2 x), \varphi(x, x), \varphi(x, 3 x), \varphi((1+2 k) x, x), \varphi((1-2 k) x, x)\right\}
\end{array}
$$

for all $x \in X$.

Proof. Using the oddness of $f$ and from (3.3), we have

$$
\left\|f(x+k y)+f(x-k y)-k^{2} f(x+y)-k^{2} f(x-y)-2\left(1-k^{2}\right) f(x)\right\| \leq \varphi(x, y)
$$

for all $x, y \in X$. Replacing $y$ by $x$ in (3.6), we get

$$
\left\|f((1+k) x)+f((1-k) x)-k^{2} f(2 x)-2\left(1-k^{2}\right) f(x)\right\| \leq \varphi(x, x)
$$


for all $x \in X$. Replacing $x$ by $2 x$ in (3.7), we get

$$
\left\|f(2(1+k) x)+f(2(1-k) x)-k^{2} f(4 x)-2\left(1-k^{2}\right) f(2 x)\right\| \leq \varphi(2 x, 2 x)
$$

for all $x \in X$. Replacing $x$ and $y$ by $2 x$ and $x$ in (3.6), respectively, we get

$$
\left\|f((2+k) x)+f((2-k) x)-k^{2} f(3 x)-k^{2} f(x)-2\left(1-k^{2}\right) f(2 x)\right\| \leq \varphi(2 x, x)
$$

for all $x \in X$. Replacing $y$ by $2 x$ in (3.6), we get

$$
\left\|f((1+2 k) x)+f((1-2 k) x)-k^{2} f(3 x)+k^{2} f(x)-2\left(1-k^{2}\right) f(x)\right\| \leq \varphi(x, 2 x)
$$

for all $x \in X$. Replacing $y$ by $3 x$ in (3.6), we get

$$
\left\|f((1+3 k) x)+f((1-3 k) x)-k^{2} f(4 x)+k^{2} f(2 x)-2\left(1-k^{2}\right) f(x)\right\| \leq \varphi(x, 3 x)
$$

for all $x \in X$. Replacing $x$ and $y$ by $(1+k) x$ and $x$ in (3.6), respectively, we have

$$
\left\|f((1+2 k) x)+f(x)-k^{2} f((2+k) x)-k^{2} f(k x)-2\left(1-k^{2}\right) f((1+k) x)\right\| \leq \varphi((1+k) x, x)
$$

for all $x \in X$. Again, replacing $x$ and $y$ by $(1-k) x$ and $x$ in (3.6), respectively, we have

$$
\left\|f(x)+f((1-2 k) x)-k^{2} f((2-k) x)+k^{2} f(k x)-2\left(1-k^{2}\right) f((1-k) x)\right\| \leq \varphi((1-k) x, x)
$$

for all $x \in X$. By (3.12) and (3.13), we have

$$
\begin{aligned}
& \| f((1+2 k) x)+f((1-2 k) x)+2 f(x)-k^{2} f((2+k) x) \\
& -k^{2} f((2-k) x)-2\left(1-k^{2}\right) f((1+k) x)-2\left(1-k^{2}\right) f((1-k) x) \| \\
& \quad \leq \max \{\varphi((1+k) x, x), \varphi((1-k) x, x)\}
\end{aligned}
$$

for all $x \in X$. Replacing $x$ and $y$ by $(1+2 k) x$ and $x$ in (3.6), respectively, we have

$$
\begin{aligned}
& \left\|f((1+3 k) x)+f((1+k) x)-k^{2} f(2(1+k) x)-k^{2} f(2 k x)-2\left(1-k^{2}\right) f((1+2 k) x)\right\| \\
& \quad \leq \varphi((1+2 k) x, x)
\end{aligned}
$$


for all $x \in X$. Again, replacing $x$ and $y$ by $(1-2 k) x$ and $x$ in (3.6), respectively, we obtain

$$
\begin{aligned}
& \left\|f((1-3 k) x)+f((1-k) x)-k^{2} f(2(1-k) x)+k^{2} f(2 k x)-2\left(1-k^{2}\right) f((1-2 k) x)\right\| \\
& \quad \leq \varphi((1-2 k) x, x)
\end{aligned}
$$

for all $x \in X$. By (3.15) and (3.16), we have

$$
\begin{aligned}
& \| f((1+3 k) x)+f((1-3 k) x)+f((1+k) x)+f((1-k) x)-k^{2} f(2(1+k) x) \\
& -k^{2} f(2(1-k) x)-2\left(1-k^{2}\right) f((1+2 k) x)-2\left(1-k^{2}\right) f((1-2 k) x) \| \\
& \quad \leq \max \{\varphi((1+2 k) x, x), \varphi((1-2 k) x, x)\}
\end{aligned}
$$

for all $x \in X$. By (3.7), (3.9), (3.10), and (3.14), we get

$$
\begin{aligned}
& \left\|\left(k^{4}-k^{2}\right)[f(3 x)-4 f(2 x)+5 f(x)]\right\| \\
& \quad \leq \max \left\{\left|2\left(1-k^{2}\right)\right| \varphi(x, x),\left|k^{2}\right| \varphi(2 x, x), \varphi(x, 2 x), \varphi((1+k) x, x), \varphi((1-k) x, x)\right\}
\end{aligned}
$$

for all $x \in X$. Hence,

$$
\begin{aligned}
& \|f(3 x)-4 f(2 x)+5 f(x)\| \\
& \quad \leq \frac{1}{\left|k^{4}-k^{2}\right|} \max \left\{\left|2\left(1-k^{2}\right)\right| \varphi(x, x),\left|k^{2}\right| \varphi(2 x, x), \varphi(x, 2 x), \varphi((1+k) x, x), \varphi((1-k) x, x)\right\}
\end{aligned}
$$

for all $x \in X$. By (3.7), (3.8), (3.10), (3.11), and (3.17), we get

$$
\begin{gathered}
\left\|\left(k^{4}-k^{2}\right)[f(4 x)-2 f(3 x)-2 f(2 x)+6 f(x)]\right\| \\
\leq \max \left\{\left|k^{2}\right| \varphi(2 x, 2 x),\left|2\left(1-k^{2}\right)\right| \varphi(x, 2 x), \varphi(x, x), \varphi(x, 3 x),\right. \\
\varphi((1+2 k) x, x), \varphi((1-2 k) x, x)\}
\end{gathered}
$$

for all $x \in X$. Hence,

$$
\begin{gathered}
\|f(4 x)-2 f(3 x)-2 f(2 x)+6 f(x)\| \\
\leq \frac{1}{\left|k^{4}-k^{2}\right|} \max \left\{\left|k^{2}\right| \varphi(2 x, 2 x),\left|2\left(1-k^{2}\right)\right| \varphi(x, 2 x), \varphi(x, x), \varphi(x, 3 x),\right. \\
\varphi((1+2 k) x, x), \varphi((1-2 k) x, x)\}
\end{gathered}
$$


for all $x \in X$. By (3.19) and (3.21), we get

$$
\begin{aligned}
& \| f(4 x)-10 f(2 x)+ 16 f(x) \| \\
& \leq \frac{1}{\left|k^{4}-k^{2}\right|} \max \left\{\left|2 k^{2}\right| \varphi(2 x, x),|2| \varphi(x, 2 x),|2| \varphi((1+k) x, x),|2| \varphi((1-k) x, x),\right. \\
&\left.\left|k^{2}\right| \varphi(2 x, 2 x), \varphi(x, x), \varphi(x, 3 x), \varphi((1+2 k) x, x), \varphi((1-2 k) x, x)\right\}
\end{aligned}
$$

for all $x \in X$. Therefore,

$$
\|f(4 x)-10 f(2 x)+16 f(x)\| \leq \tilde{\varphi}(x),
$$

where

$$
\begin{aligned}
\tilde{\varphi}(x):=\frac{1}{\left|k^{4}-k^{2}\right|} \max \left\{\left|2 k^{2}\right| \varphi(2 x, x),|2| \varphi(x, 2 x),|2| \varphi((1+k) x, x),|2| \varphi((1-k) x, x),\right. \\
\\
\left.\left|k^{2}\right| \varphi(2 x, 2 x), \varphi(x, x), \varphi(x, 3 x), \varphi((1+2 k) x, x), \varphi((1-2 k) x, x)\right\}
\end{aligned}
$$

for all $x \in X$.

Consider the set

$$
\Omega:=\{g \mid g: X \longrightarrow Y\},
$$

and introduce the generalized metric on $\Omega$,

$$
d(g, h)=\inf \{\alpha>0 \mid\|g(x)-h(x)\| \leq \alpha \tilde{\varphi}(x) \forall x \in X\} .
$$

It is easy to show that $(\Omega, d)$ is a complete generalized metric space (see [19, Lemma 2.1]).

Define a function $J: \Omega \rightarrow \Omega$ by $J g(x)=2 g(x / 2)$ for all $x \in X$. Let $g, h \in \Omega$ be given such that $d(g, h)<\beta$; by the definition,

$$
\|g(x)-h(x)\| \leq \beta \tilde{\varphi}(x), \quad \forall x \in X
$$

Hence,

$$
\|J g(x)-J h(x)\|=|2||| g\left(\frac{x}{2}\right)-h\left(\frac{x}{2}\right) \| \leq|2| \beta \tilde{\varphi}\left(\frac{x}{2}\right) \leq|2| \beta \frac{L}{|2|} \tilde{\varphi}(x)=L \beta \tilde{\varphi}(x)
$$

for all $x \in X$. By definition, $d(J g, J h) \leq L \beta$. Therefore,

$$
d(J g, J h) \leq L d(g, h), \quad \forall g, h \in \Omega .
$$


This means that $J$ is a strictly contractive self-mapping of $\Omega$ with Lipschitz constant $L$.

Now, let $g: X \rightarrow Y$ be the function defined by $g(x):=f(2 x)-8 f(x)$ for each $x \in X$. By (3.23), we get

$$
\|g(2 x)-2 g(x)\| \leq \tilde{\varphi}(x)
$$

for all $x \in X$. Replacing $x$ by $x / 2$ in (3.30), we obtain

$$
\left\|g(x)-2 g\left(\frac{x}{2}\right)\right\| \leq \tilde{\varphi}\left(\frac{x}{2}\right) \leq \frac{L}{|2|} \tilde{\varphi}(x)
$$

for all $x \in X$. Hence $d(g, J g) \leq L /|2|$, and therefore, by Theorem 2.4, $J$ has a unique fixed point $A: X \rightarrow Y$ in the set $\Delta=\{h \in \Omega: d(g, h)<\infty\}$. This implies that $A(x / 2)=(1 / 2) A(x)$ and

$$
A(x):=\lim _{n \rightarrow \infty} J^{n} g(x)=\lim _{n \rightarrow \infty} 2^{n} g\left(\frac{x}{2^{n}}\right)
$$

for all $x \in X$. Since $g: X \rightarrow Y$ is odd, $A: X \rightarrow Y$ is an odd function. Moreover,

$$
d(g, A) \leq \frac{1}{1-L} d(g, J g) \leq \frac{L}{|2|-|2| L}
$$

This implies that inequality (3.4) holds.

Replacing $x, y$ by $2^{-n} x, 2^{-n} y$, respectively, in (3.3), we have

$$
\left\|2^{n} D g\left(2^{-n} x, 2^{-n} y\right)\right\| \leq\left|2^{n}\right| \max \left\{\tilde{\varphi}\left(\frac{2 x}{2^{n}}, \frac{2 y}{2^{n}}\right),|8| \tilde{\varphi}\left(\frac{x}{2^{n}}, \frac{y}{2^{n}}\right)\right\}
$$

for all $x, y \in X$ and $n \in \mathbb{N}$. Hence,

$$
\left\|2^{n} D g\left(2^{-n} x, 2^{-n} y\right)\right\| \leq\left|2^{n}\right| \frac{L^{n}}{|2|^{n}} \max \{\tilde{\varphi}(2 x, 2 y),|8| \tilde{\varphi}(x, y)\}=L^{n} \max \{\tilde{\varphi}(2 x, 2 y),|8| \tilde{\varphi}(x, y)\}
$$

for all $x, y \in X$ and $n \in \mathbb{N}$. So $\|D A(x, y)\|=0$ for all $x, y \in X$. By Theorem 2.2 of [10], the function $x \rightarrow A(2 x)-8 A(x)$ is additive. Hence, $A(2 x)=2 A(x)$ implies that $A$ is an additive function.

To prove the uniqueness assertion, let us assume that there exists an additive function $T: X \rightarrow Y$ which satisfies (3.4). Then, $T$ is a fixed point of $J$ in $\Delta$. However, by Theorem 2.4, $J$ has only one fixed point in $\Delta$, and hence $A=T$. This completes the proof.

Remark 3.2. We observe that in case $k=2$, (1.5) yields the AQCQ-functional (1.4). Therefore, Theorem 3.1 is a generalized version of the theorem for AQCQ-functional equations (see [26, Theorem 2.4]). 
Corollary 3.3. Let $\delta>0,0<r<1,|2|<1$, and let $f: X \rightarrow Y$ be an odd function satisfying

$$
\|D f(x, y)\| \leq \delta\left(\|x\|^{r}+\|y\|^{r}\right)
$$

for all $x, y \in X$. Then, there exists a unique additive function $A: X \rightarrow Y$ such that

$$
\|f(2 x)-8 f(x)-A(x)\| \leq \frac{2 \delta\|x\|^{r}}{\left|k^{4}-k^{2}\right|\left(|2|^{r}-|2|\right)}
$$

for all $x \in X$.

Proof. Let $\varphi: X \times X \rightarrow[0, \infty)$ be defined by $\varphi(x, y)=\delta\left(\|x\|^{r}+\|y\|^{r}\right)$ for all $x, y \in X$. Then, the corollary is followed from Theorem 3.1 by $L=|2| /|2|^{r}<1$.

Corollary 3.4. Let $\delta>0,|2|<1$, and let $f: X \rightarrow Y$ be an odd function satisfying

$$
\|D f(x, y)\| \leq \delta\left[\|x\|^{r}\|y\|^{s}+\left(\|x\|^{r+s}+\|y\|^{r+s}\right)\right] \quad(x, y \in X),
$$

where $r$, s are nonnegative real numbers such that $\lambda:=r+s \in(0,1)$. Then, there exists a unique additive function $A: X \rightarrow Y$ such that

$$
\|f(2 x)-8 f(x)-A(x)\| \leq \frac{3 \delta\|x\|^{\curlywedge}}{\left|k^{4}-k^{2}\right|\left(|2|^{\lambda}-|2|\right)}
$$

for all $x \in X$.

Proof. Let $\varphi: X \times X \rightarrow[0, \infty)$ be defined by $\varphi(x, y)=\delta\left[\|x\|^{r}\|y\|^{s}+\left(\|x\|^{r+s}+\|y\|^{r+s}\right)\right]$ for all $x, y \in X$. Then, the corollary is followed from Theorem 3.1 by $L=|2| /|2|^{\lambda}<1$.

Theorem 3.5. Let $\varphi: X \times X \rightarrow[0, \infty)$ be a function such that there exists an $L<1$ with

$$
\varphi(x, y) \leq|2| L \varphi\left(\frac{x}{2}, \frac{y}{2}\right)
$$

for all $x, y \in X$. Let $f: X \rightarrow Y$ be an odd function satisfying

$$
\|D f(x, y)\| \leq \varphi(x, y)
$$

for all $x, y \in X$. Then, there exists a unique additive function $A: X \rightarrow Y$ such that

$$
\|f(2 x)-8 f(x)-A(x)\| \leq \frac{1}{|2|-|2| L} \tilde{\varphi}(x)
$$

for all $x \in X$, where $\tilde{\varphi}(x)$ is defined as in Theorem 3.1. 
Proof. From (3.30) and (3.40), we get

$$
\left\|g(x)-\frac{1}{2} g(2 x)\right\| \leq \frac{1}{|2|} \tilde{\varphi}(x)
$$

for all $x \in X$. The rest of the proof is similar to the proof of Theorem 3.1.

Theorem 3.6. Let $\varphi: X \times X \rightarrow[0, \infty)$ be a function such that there exists an $L<1$ with

$$
\varphi(x, y) \leq \frac{L}{|8|} \varphi(2 x, 2 y)
$$

for all $x, y \in X$. Let $f: X \rightarrow Y$ be an odd function satisfying

$$
\|D f(x, y)\| \leq \varphi(x, y)
$$

for all $x, y \in X$. Then there exists a unique cubic function $C: X \rightarrow Y$ such that

$$
\|f(2 x)-2 f(x)-C(x)\| \leq \frac{L}{|8|-|8| L} \tilde{\varphi}(x)
$$

for all $x \in X$, where $\tilde{\varphi}(x)$ is defined as in Theorem 3.1.

Proof. Letting $h: X \rightarrow Y$ be the function defined by $h(x):=f(2 x)-2 f(x)$ for each $x \in X$, then by (3.23), (3.24), and (3.44), we get

$$
\left\|h(x)-8 h\left(\frac{x}{2}\right)\right\| \leq \tilde{\varphi}\left(\frac{x}{2}\right) \leq \frac{L}{|8|} \tilde{\varphi}(x)
$$

for all $x \in X$. The rest of the proof is similar to the proof of Theorem 3.1.

Corollary 3.7. Let $\delta>0,0<r<3,|2|<1$, and let $f: X \rightarrow Y$ be an odd function satisfying

$$
\|D f(x, y)\| \leq \delta\left(\|x\|^{r}+\|y\|^{r}\right)
$$

for all $x, y \in X$. Then, there exists a unique cubic function $C: X \rightarrow Y$ such that

$$
\|f(2 x)-2 f(x)-C(x)\| \leq \frac{2 \delta\|x\|^{r}}{\left|k^{4}-k^{2}\right|\left(|2|^{r}-|8|\right)}
$$

for all $x \in X$.

Proof. Let $\varphi: X \times X \rightarrow[0, \infty)$ be defined by $\varphi(x, y)=\delta\left(\|x\|^{r}+\|y\|^{r}\right)$ for all $x, y \in X$. Then, the corollary is followed from Theorem 3.6 by $L=|8| /|2|^{r}<1$. 
Corollary 3.8. Let $\delta>0,|2|<1$ and $f: X \rightarrow Y$ be an odd function satisfying

$$
\|D f(x, y)\| \leq \delta\left[\|x\|^{r}\|y\|^{s}+\left(\|x\|^{r+s}+\|y\|^{r+s}\right)\right] \quad(x, y \in X),
$$

where $r$, s are nonnegative real numbers such that $\lambda:=r+s \in(0,3)$. Then, there exists a unique cubic function $C: X \rightarrow Y$ such that

$$
\|f(2 x)-2 f(x)-C(x)\| \leq \frac{3 \delta\|x\|^{\lambda}}{\left|k^{4}-k^{2}\right|\left(|2|^{\lambda}-|8|\right)}
$$

for all $x \in X$

Proof. Let $\varphi: X \times X \rightarrow[0, \infty)$ be defined by $\varphi(x, y)=\delta\left[\|x\|^{r}\|y\|^{s}+\left(\|x\|^{r+s}+\|y\|^{r+s}\right)\right]$ for all $x, y \in X$. Then, the corollary is followed from Theorem 3.6 by $L=|8| /|2|^{\lambda}<1$.

Theorem 3.9. Let $\varphi: X \times X \rightarrow[0, \infty)$ be a function such that there exists an $L<1$ with

$$
\varphi(x, y) \leq|8| L \varphi\left(\frac{x}{2}, \frac{y}{2}\right)
$$

for all $x, y \in X$. Let $f: X \rightarrow Y$ be an odd function satisfying

$$
\|D f(x, y)\| \leq \varphi(x, y)
$$

for all $x, y \in X$. Then, there exists a unique cubic function $C: X \rightarrow Y$ such that

$$
\|f(2 x)-2 f(x)-C(x)\| \leq \frac{L}{|8|-|8| L} \tilde{\varphi}(x)
$$

for all $x \in X$, where $\tilde{\varphi}(x)$ is defined as in Theorem 3.1.

Proof. Let $h: X \rightarrow Y$ be the function defined by $h(x):=f(2 x)-2 f(x)$ for each $x \in X$. By (3.23), we get

$$
\left\|h(x)-\frac{1}{8} h(2 x)\right\| \leq \frac{1}{|8|} \tilde{\varphi}(x)
$$

for all $x \in X$. The rest of the proof is similar to the proof of Theorem 3.1.

Theorem 3.10. Let $\varphi: X \times X \rightarrow[0, \infty)$ be a function such that there exists an $L<1$ with

$$
\varphi(x, y) \leq \frac{L}{|4|} \varphi(2 x, 2 y)
$$


for all $x, y \in X$. Let $f: X \rightarrow Y$ be an even function with $f(0)=0$ satisfying

$$
\|D f(x, y)\| \leq \varphi(x, y)
$$

for all $x, y \in X$. Then, there exists a unique quadratic function $B: X \rightarrow Y$ such that

$$
\|f(2 x)-16 f(x)-B(x)\| \leq \frac{L}{|4|-|4| L} \Phi(x)
$$

for all $x \in X$, where

$$
\Phi(x):=\frac{1}{\left|k^{4}-k^{2}\right|} \max \left\{\left|12\left(1-k^{2}\right)\right| \varphi(0, x),\left|12 k^{2}\right| \varphi(x, x),|6| \varphi(0,2 x),|12| \varphi(k x, x)\right\}
$$

for all $x \in X$.

Proof. Using the evenness of $f$ and from (3.57), we have

$$
\begin{aligned}
& \| f(x+k y)+f(x-k y)-k^{2} f(x+y)-k^{2} f(x-y)-2\left(1-k^{2}\right) f(x) \\
& -\frac{k^{4}-k^{2}}{12}[2 f(2 y)-8 f(y)] \| \leq \varphi(x, y)
\end{aligned}
$$

for all $x, y \in X$. Interchanging $x$ and $y$ in (3.60), we get

$$
\begin{aligned}
& \| f(k x+y)+f(k x-y)-k^{2} f(x+y)-k^{2} f(x-y)-2\left(1-k^{2}\right) f(y) \\
& -\frac{k^{4}-k^{2}}{12}[2 f(2 x)-8 f(x)] \| \leq \varphi(y, x)
\end{aligned}
$$

for all $x, y \in X$. Letting $y=0$ in (3.61), we get

$$
\left\|2 f(k x)-2 k^{2} f(x)-\frac{k^{4}-k^{2}}{12}[2 f(2 x)-8 f(x)]\right\| \leq \varphi(0, x)
$$

for all $x \in X$. Putting $y=x$ in (3.61), we have

$$
\left\|f((k+1) x)+f((k-1) x)-k^{2} f(2 x)-2\left(1-k^{2}\right) f(x)-\frac{k^{4}-k^{2}}{12}[2 f(2 x)-8 f(x)]\right\| \leq \varphi(x, x)
$$


for all $x \in X$. Replacing $x$ by $2 x$ in (3.62), we get

$$
\left\|2 f(2 k x)-2 k^{2} f(2 x)-\frac{k^{4}-k^{2}}{12}[2 f(4 x)-8 f(2 x)]\right\| \leq \varphi(0,2 x)
$$

for all $x \in X$. Letting $y=k x$ in (3.61), we get

$$
\begin{aligned}
& \| f(2 k x)-k^{2} f((1+k) x)-k^{2} f((1-k) x)-2\left(1-k^{2}\right) f(k x) \\
& -\frac{k^{4}-k^{2}}{12}[2 f(2 x)-8 f(x)] \| \leq \varphi(k x, x)
\end{aligned}
$$

for all $x \in X$. By (3.62)-(3.65), we obtain

$$
\begin{aligned}
& \left\|\left(k^{4}-k^{2}\right)[f(4 x)-20 f(2 x)+64 f(x)]\right\| \\
& \quad \leq \max \left\{\left|12\left(1-k^{2}\right)\right| \varphi(0, x),\left|12 k^{2}\right| \varphi(x, x),|6| \varphi(0,2 x),|12| \varphi(k x, x)\right\}
\end{aligned}
$$

for all $x \in X$. Hence,

$$
\begin{aligned}
& \|f(4 x)-20 f(2 x)+64 f(x)\| \\
& \leq \frac{1}{\left|k^{4}-k^{2}\right|} \max \left\{\left|12\left(1-k^{2}\right)\right| \varphi(0, x),\left|12 k^{2}\right| \varphi(x, x),|6| \varphi(0,2 x),|12| \varphi(k x, x)\right\}
\end{aligned}
$$

for all $x \in X$. From (3.67), we get

$$
\|f(4 x)-20 f(2 x)+64 f(x)\| \leq \Phi(x)
$$

for all $x \in X$, where

$$
\Phi(x):=\frac{1}{\left|k^{4}-k^{2}\right|} \max \left\{\left|12\left(1-k^{2}\right)\right| \varphi(0, x),\left|12 k^{2}\right| \varphi(x, x),|6| \varphi(0,2 x),|12| \varphi(k x, x)\right\}
$$

for all $x \in X$.

Consider the set

$$
\Omega:=\{g \mid g: X \longrightarrow Y\},
$$

and introduce the generalized metric on $\Omega$,

$$
d(g, h)=\inf \{\alpha>0 \mid\|g(x)-h(x)\| \leq \alpha \Phi(x) \forall x \in X\} .
$$


It is easy to show that $(\Omega, d)$ is a complete generalized metric space (see [19, Lemma 2.1]).

Define a function $J: \Omega \rightarrow \Omega$ by $J g(x)=4 g(x / 2)$ for all $x \in X$. Similar to the proof of Theorem 3.1, one can prove that $J$ is a strictly contractive self-mapping of $\Omega$ with Lipschitz constant $L$.

Now, let $g: X \rightarrow Y$ be the function defined by $g(x):=f(2 x)-16 f(x)$ for each $x \in X$. By (3.68), we get

$$
\|g(2 x)-4 g(x)\| \leq \Phi(x)
$$

for all $x \in X$. Replacing $x$ by $x / 2$, we obtain

$$
\left\|g(x)-4 g\left(\frac{x}{2}\right)\right\| \leq \Phi\left(\frac{x}{2}\right) \leq \frac{L}{|4|} \Phi(x)
$$

for all $x \in X$. Hence, $d(g, J g) \leq L /|4|$, and therefore, by Theorem 2.4, $J$ has a unique fixed point $B: X \rightarrow Y$ in the set $\Delta=\{h \in \Omega: d(g, h)<\infty\}$. This implies that $B(x / 2)=(1 / 4) B(x)$ and

$$
B(x):=\lim _{n \rightarrow \infty} J^{n} g(x)=\lim _{n \rightarrow \infty} 4^{n} g\left(\frac{x}{2^{n}}\right)
$$

for all $x \in X$. Since $g: X \rightarrow Y$ is even, $B: X \rightarrow Y$ is an even function. Moreover,

$$
d(g, B) \leq \frac{1}{1-L} d(g, J g) \leq \frac{L}{|4|-|4| L}
$$

This implies that inequality (3.58) holds. Similar to the proof of Theorem 3.1, by Theorem 2.1 of [10], the function $x \rightarrow B(2 x)-16 B(x)$ is quadratic. Hence, $B(2 x)=4 B(x)$ implies that $B$ is a quadratic function.

The rest of the proof is similar to the proof of Theorem 3.1.

Corollary 3.11. Let $\delta>0,0<r<2,|2|<1$, and let $f: X \rightarrow Y$ be an even function with $f(0)=0$ satisfying

$$
\|D f(x, y)\| \leq \delta\left(\|x\|^{r}+\|y\|^{r}\right)
$$

for all $x, y \in X$. Then, there exists a unique quadratic function $B: X \rightarrow Y$ such that

$$
\begin{aligned}
& \|f(2 x)-16 f(x)-B(x)\| \\
& \quad \leq \max \left\{\left|12\left(1-k^{2}\right)\right|, 2\left|12 k^{2}\right|,|6||2|^{r},|12|\left(|k|^{r}+1\right)\right\} \frac{\delta\|x\|^{r}}{\left|k^{4}-k^{2}\right|\left(|2|^{r}-|4|\right)}
\end{aligned}
$$

for all $x \in X$.

Proof. Let $\varphi: X \times X \rightarrow[0, \infty)$ be defined by $\varphi(x, y)=\delta\left(\|x\|^{r}+\|y\|^{r}\right)$ for all $x, y \in X$. Then, the corollary is followed from Theorem 3.10 by $L=|4| /|2|^{r}<1$. 
Corollary 3.12. Let $\delta>0,|2|<1$, and let $f: X \rightarrow Y$ be an even function with $f(0)=0$ satisfying

$$
\|D f(x, y)\| \leq \delta\left[\|x\|^{r}\|y\|^{s}+\left(\|x\|^{r+s}+\|y\|^{r+s}\right)\right] \quad(x, y \in X),
$$

where $r$, s are nonnegative real numbers such that $\lambda:=r+s \in(0,2)$. Then, there exists a unique quadratic function $B: X \rightarrow Y$ such that

$$
\begin{aligned}
& \|f(2 x)-16 f(x)-B(x)\| \\
& \quad \leq \max \left\{\left|12\left(1-k^{2}\right)\right|, 3\left|12 k^{2}\right|,|6||2|^{\lambda},|12|\left(|k|^{r}+|k|^{\lambda}+1\right)\right\} \frac{\delta\|x\|^{r}}{\left|k^{4}-k^{2}\right|\left(|2|^{r}-|4|\right)}
\end{aligned}
$$

for all $x \in X$.

Proof. Let $\varphi: X \times X \rightarrow[0, \infty)$ be defined by $\varphi(x, y)=\delta\left[\|x\|^{r}\|y\|^{s}+\left(\|x\|^{r+s}+\|y\|^{r+s}\right)\right]$ for all $x, y \in X$. Then, the corollary is followed from Theorem 3.10 by $L=|4| /|2|^{\lambda}<1$.

Theorem 3.13. Let $\varphi: X \times X \rightarrow[0, \infty)$ be a function such that there exists an $L<1$ with

$$
\varphi(x, y) \leq|4| L \varphi\left(\frac{x}{2}, \frac{y}{2}\right)
$$

for all $x, y \in X$. Let $f: X \rightarrow Y$ be an even function with $f(0)=0$ satisfying

$$
\|D f(x, y)\| \leq \varphi(x, y)
$$

for all $x, y \in X$. Then, there exists a unique quadratic function $B: X \rightarrow Y$ such that

$$
\|f(2 x)-16 f(x)-B(x)\| \leq \frac{1}{|4|-|4| L} \Phi(x)
$$

for all $x \in X$, where $\Phi(x)$ is defined as in Theorem 3.10.

Proof. Let $g: X \rightarrow Y$ be the function defined by $g(x):=f(2 x)-16 f(x)$ for each $x \in X$. By (3.68), we get

$$
\left\|g(x)-\frac{1}{8} g(2 x)\right\| \leq \frac{1}{|8|} \tilde{\varphi}(x)
$$

for all $x \in X$. The rest of the proof is similar to the proof of Theorem 3.10.

Theorem 3.14. Let $\varphi: X \times X \rightarrow[0, \infty)$ be a function such that there exists an $L<1$ with

$$
\varphi(x, y) \leq \frac{L}{|16|} \varphi(2 x, 2 y)
$$


for all $x, y \in X$. Let $f: X \rightarrow Y$ be an even function with $f(0)=0$ satisfying

$$
\|D f(x, y)\| \leq \varphi(x, y)
$$

for all $x, y \in X$. Then, there exists a unique quartic function $Q: X \rightarrow Y$ such that

$$
\|f(2 x)-4 f(x)-Q(x)\| \leq \frac{L}{|16|-|16| L} \Phi(x)
$$

for all $x \in X$, where $\Phi(x)$ is defined as in Theorem 3.10.

Proof. Letting $h: X \rightarrow Y$ be the function defined by $h(x):=f(2 x)-4 f(x)$ for each $x \in X$, then by (3.68), (3.69), and (3.84), we get

$$
\left\|h(x)-16 h\left(\frac{x}{2}\right)\right\| \leq \Phi\left(\frac{x}{2}\right) \leq \frac{L}{|16|} \Phi(x)
$$

for all $x \in X$.

The rest of the proof is similar to the proof of Theorem 3.1.

Corollary 3.15. Let $\delta>0,0<r<4,|2|<1$, and let $f: X \rightarrow Y$ be an even function with $f(0)=0$ satisfying

$$
\|D f(x, y)\| \leq \delta\left(\|x\|^{r}+\|y\|^{r}\right)
$$

for all $x, y \in X$. Then, there exists a unique quartic function $Q: X \rightarrow Y$ such that

$$
\begin{aligned}
& \|f(2 x)-4 f(x)-Q(x)\| \\
& \leq \max \left\{\left|12\left(1-k^{2}\right)\right|, 2\left|12 k^{2}\right|,|6||2|^{r},|12|\left(|k|^{r}+1\right)\right\} \frac{\delta\|x\|^{r}}{\left|k^{4}-k^{2}\right|\left(|2|^{r}-|16|\right)}
\end{aligned}
$$

for all $x \in X$.

Proof. Let $\varphi: X \times X \rightarrow[0, \infty)$ be defined by $\varphi(x, y)=\delta\left(\|x\|^{r}+\|y\|^{r}\right)$ for all $x, y \in X$. Then, the corollary is followed from Theorem 3.14 by $L=|16| /|2|^{r}<1$. 
Corollary 3.16. Let $\delta>0,|2|<1$, and let $f: X \rightarrow Y$ be an even function with $f(0)=0$ satisfying

$$
\|D f(x, y)\| \leq \delta\left[\|x\|^{r}\|y\|^{s}+\left(\|x\|^{r+s}+\|y\|^{r+s}\right)\right] \quad(x, y \in X),
$$

where $r$, s are nonnegative real numbers such that $\lambda:=r+s \in(0,4)$. Then, there exists a unique quartic function $Q: X \rightarrow Y$ such that

$$
\begin{aligned}
& \|f(2 x)-4 f(x)-Q(x)\| \\
& \quad \leq \max \left\{\left|12\left(1-k^{2}\right)\right|, 3\left|12 k^{2}\right|,|6||2|^{\curlywedge},|12|\left(|k|^{r}+|k|^{\curlywedge}+1\right)\right\} \frac{\delta\|x\|^{r}}{\left|k^{4}-k^{2}\right|\left(|2|^{r}-|16|\right)}
\end{aligned}
$$

for all $x \in X$.

Proof. Let $\varphi: X \times X \rightarrow[0, \infty)$ be defined by $\varphi(x, y)=\delta\left[\|x\|^{r}\|y\|^{s}+\left(\|x\|^{r+s}+\|y\|^{r+s}\right)\right]$ for all $x, y \in X$. Then, the corollary is followed from Theorem 3.14 by $L=|16| /|2|^{\lambda}<1$.

Theorem 3.17. Let $\varphi: X \times X \rightarrow[0, \infty)$ be a function such that there exists an $L<1$ with

$$
\varphi(x, y) \leq|16| L \varphi\left(\frac{x}{2}, \frac{y}{2}\right)
$$

for all $x, y \in X$. Let $f: X \rightarrow Y$ be an even function with $f(0)=0$ satisfying

$$
\|D f(x, y)\| \leq \varphi(x, y)
$$

for all $x, y \in X$. Then, there exists a unique quartic function $Q: X \rightarrow Y$ such that

$$
\|f(2 x)-4 f(x)-Q(x)\| \leq \frac{1}{|16|-|16| L} \Phi(x)
$$

for all $x \in X$, where $\Phi(x)$ is defined as in Theorem 3.10.

Proof. Let $h: X \rightarrow Y$ be the function defined by $h(x):=f(2 x)-4 f(x)$ for each $x \in X$. By (3.68), we get

$$
\left\|h(x)-\frac{1}{16} h(2 x)\right\| \leq \frac{1}{|16|} \Phi(x)
$$

for all $x \in X$. The rest of the proof is similar to the proof of Theorem 3.10.

Theorem 3.18. Let $\varphi: X \times X \rightarrow[0, \infty)$ be a function such that there exists an $L<1$ with

$$
\varphi(x, y) \leq \frac{L}{|2|} \varphi(2 x, 2 y)
$$


for all $x, y \in X$. Let $f: X \rightarrow Y$ be a function with $f(0)=0$ satisfying

$$
\|D f(x, y)\| \leq \varphi(x, y)
$$

for all $x, y \in X$. Then, there exist an additive function $A: X \rightarrow Y$, a quadratic function $B: X \rightarrow Y$, a cubic function $C: X \rightarrow Y$, and a quartic function $Q: X \rightarrow Y$ such that

$$
\begin{aligned}
&\|f(x)-A(x)-B(x)-C(x)-Q(x)\| \\
& \leq \frac{L}{|96|\left|k^{4}-k^{2}\right|(1-L)} \\
& \max \left\{\left|2 k^{2}\right| \varphi(2 x, x),|2| \varphi(x, 2 x),|2| \varphi((1+k) x, x),|2| \varphi((1-k) x, x),\right. \\
& \quad\left|k^{2}\right| \varphi(2 x, 2 x), \varphi(x, x), \varphi(x, 3 x), \varphi((1+2 k) x, x), \varphi((1-2 k) x, x), \\
&\left|2 k^{2}\right| \varphi(-2 x,-x),|2| \varphi(-x,-2 x),|2| \varphi(-(1+k) x,-x), \\
&|2| \varphi(-(1-k) x,-x),\left|k^{2}\right| \varphi(-2 x,-2 x), \varphi(-x,-x), \varphi(-x,-3 x), \\
& \varphi(-(1+2 k) x,-x), \varphi(-(1-2 k) x,-x),\left|3\left(1-k^{2}\right)\right| \varphi(0, x), \\
& \frac{|3|}{|2|} \varphi(0,2 x),|3| \varphi(k x, x),\left|3\left(1-k^{2}\right)\right| \varphi(0,-x), \\
&\left.\frac{|3|}{|2|} \varphi(0,-2 x),|3| \varphi(-k x,-x)\right\}
\end{aligned}
$$

for all $x \in X$.

Proof. Let $f_{o}(x)=(1 / 2)[f(x)-f(-x)]$, and let $f_{e}(x)=(1 / 2)[f(x)+f(-x)]$ for all $x \in X$. Then, $f_{e}(0)=0, f_{o}$ is odd, and $f_{e}$ is even. Hence

$$
\begin{aligned}
\left\|D f_{o}(x, y)\right\| & =\frac{1}{|2|}\|D f(x, y)-D f(-x,-y)\| \\
& \leq \frac{1}{|2|} \max \{\|D f(x, y)\|,\|D f(-x,-y)\|\} \\
& \leq \frac{1}{|2|} \max \{\varphi(x, y), \varphi(-x,-y)\}, \\
\left\|D f_{e}(x, y)\right\| & =\frac{1}{|2|}\|D f(x, y)+D f(-x,-y)\| \\
& \leq \frac{1}{|2|} \max \{\|D f(x, y)\|,\|D f(-x,-y)\|\} \\
& \leq \frac{1}{|2|} \max \{\varphi(x, y), \varphi(-x,-y)\}
\end{aligned}
$$


for all $x, y \in X$. Putting $\varphi_{1}(x, y)=(1 /|2|) \max \{\varphi(x, y), \varphi(-x,-y)\}$ for all $x, y \in X$. Thus, from Theorems 3.1 and 3.6, there exist a unique additive function $A_{1}: X \rightarrow Y$ and a unique cubic function $C_{1}: X \rightarrow Y$ such that

$$
\begin{aligned}
\left\|f_{o}(2 x)-8 f_{o}(x)-A_{1}(x)\right\| & \leq \frac{L}{|2|-|2| L} \tilde{\varphi}_{1}(x) \\
\left\|f_{o}(2 x)-2 f_{o}(x)-C_{1}(x)\right\| & \leq \frac{L}{|8|-|8| L} \widetilde{\varphi}_{1}(x)
\end{aligned}
$$

for all $x \in X$, where $\tilde{\varphi}_{1}(x)$ is defined as in Theorem 3.1. Similarly, from Theorems 3.10 and 3.14, there exist a unique quadratic function $B_{1}: X \rightarrow Y$ and a unique quartic function $Q_{1}: X \rightarrow Y$ such that

$$
\begin{aligned}
& \left\|f_{e}(2 x)-16 f_{e}(x)-B_{1}(x)\right\| \leq \frac{L}{|4|-|4| L} \Phi_{1}(x) \\
& \left\|f_{e}(2 x)-4 f_{e}(x)-Q_{1}(x)\right\| \leq \frac{L}{|16|-|16| L} \Phi_{1}(x)
\end{aligned}
$$

for all $x \in X$, where $\Phi_{1}(x)$ is defined as in Theorem 3.10. Defining $A(x)=-A_{1}(x) / 6, C(x)=$ $C_{1}(x) / 6, B(x)=-B_{1}(x) / 12$ and $Q(x)=Q_{1}(x) / 12$, by $f(x)=f_{o}(x)+f_{e}(x),(3.100)$, and (3.101), it follows that

$$
\begin{aligned}
\| f(x) & -A(x)-B(x)-C(x)-Q(x) \| \\
= & \left\|\left[f_{o}(x)-A(x)-C(x)\right]+\left[f_{e}(x)-B(x)-Q(x)\right]\right\| \\
\leq & \max \left\{\left\|f_{o}(x)-A(x)-C(x)\right\|,\left\|f_{e}(x)-B(x)-Q(x)\right\|\right\} \\
\leq & \max \left\{\frac{L}{|12|(1-L)} \tilde{\varphi}_{1}(x), \frac{L}{|48|(1-L)} \tilde{\varphi}_{1}(x), \frac{L}{|48|(1-L)} \Phi_{1}(x), \frac{L}{|192|(1-L)} \Phi_{1}(x)\right\} \\
\leq & \max \left\{\frac{L}{|48|(1-L)} \tilde{\varphi}_{1}(x), \frac{L}{|192|(1-L)} \Phi_{1}(x)\right\} \\
= & \frac{L}{|96|\left|k^{4}-k^{2}\right|(1-L)} \\
& \times \max \left\{\left|2 k^{2}\right| \varphi(2 x, x),|2| \varphi(x, 2 x),|2| \varphi((1+k) x, x),|2| \varphi((1-k) x, x),\right. \\
& \left|k^{2}\right| \varphi(2 x, 2 x), \varphi(x, x), \varphi(x, 3 x), \varphi((1+2 k) x, x), \varphi((1-2 k) x, x), \\
& \left|2 k^{2}\right| \varphi(-2 x,-x),|2| \varphi(-x,-2 x),|2| \varphi(-(1+k) x,-x), \\
& |2| \varphi(-(1-k) x,-x),\left|k^{2}\right| \varphi(-2 x,-2 x), \varphi(-x,-x), \varphi(-x,-3 x), \\
& \varphi(-(1+2 k) x,-x), \varphi(-(1-2 k) x,-x),\left|3\left(1-k^{2}\right)\right| \varphi(0, x),
\end{aligned}
$$




$$
\begin{aligned}
& \frac{|3|}{|2|} \varphi(0,2 x),|3| \varphi(k x, x),\left|3\left(1-k^{2}\right)\right| \varphi(0,-x), \\
& \left.\frac{|3|}{|2|} \varphi(0,-2 x),|3| \varphi(-k x,-x)\right\}
\end{aligned}
$$

for all $x \in X$.

Corollary 3.19. Let $\delta>0,0<r<1,|2|<1$, and let $f: X \rightarrow Y$ be a function with $f(0)=0$ satisfying

$$
\|D f(x, y)\| \leq \delta\left(\|x\|^{r}+\|y\|^{r}\right)
$$

for all $x, y \in X$. Then, there exist an additive function $A: X \rightarrow Y$, a quadratic function $B: X \rightarrow Y$, a cubic function $C: X \rightarrow Y$, and a quartic function $Q: X \rightarrow Y$ such that

$$
\|f(x)-A(x)-B(x)-C(x)-Q(x)\| \leq \frac{\delta \max \left\{2|2|,|3||2|^{r}\right\}}{|96|\left|k^{4}-k^{2}\right|\left(|2|^{r}-|2|\right)}\|x\|^{r}
$$

for all $x \in X$.

Proof. Let $\varphi: X \times X \rightarrow[0, \infty)$ be defined by $\varphi(x, y)=\delta\left(\|x\|^{r}+\|y\|^{r}\right)$ for all $x, y \in X$. Then, the corollary is followed from Theorem 3.18 by $L=|2| /|2|^{r}<1$.

Corollary 3.20. Let $\delta>0,|2|<1$, and let $f: X \rightarrow Y$ be a function with $f(0)=0$ satisfying

$$
\|D f(x, y)\| \leq \delta\left[\|x\|^{r}\|y\|^{s}+\left(\|x\|^{r+s}+\|y\|^{r+s}\right)\right] \quad(x, y \in X),
$$

where $r$, s are nonnegative real numbers such that $\lambda:=r+s \in(0,1)$. Then, there exist an additive function $A: X \rightarrow Y$, a quadratic function $B: X \rightarrow Y$, a cubic function $C: X \rightarrow Y$, and a quartic function $Q: X \rightarrow Y$ such that

$$
\|f(x)-A(x)-B(x)-C(x)-Q(x)\| \leq \frac{\delta \max \left\{3|2|,|3|\left(|2|^{S}+|2|^{\curlywedge}\right)\right\}}{|96|\left|k^{4}-k^{2}\right|\left(|2|^{\curlywedge}-|2|\right)}\|x\|^{\curlywedge}
$$

for all $x \in X$.

Proof. Let $\varphi: X \times X \rightarrow[0, \infty)$ be defined by $\varphi(x, y)=\delta\left[\|x\|^{r}\|y\|^{s}+\left(\|x\|^{r+s}+\|y\|^{r+s}\right)\right]$ for all $x, y \in X$. Then, the corollary is followed from Theorem 3.18 by $L=|2| /|2|^{\lambda}<1$.

Similar to Theorem 3.18, one can prove the following result.

Theorem 3.21. Let $\varphi: X \times X \rightarrow[0, \infty)$ be a function such that there exists an $L<1$ with

$$
\varphi(x, y) \leq|16| L \varphi\left(\frac{x}{2}, \frac{y}{2}\right)
$$


for all $x, y \in X$. Let $f: X \rightarrow Y$ be a function with $f(0)=0$ satisfying

$$
\|D f(x, y)\| \leq \varphi(x, y)
$$

for all $x, y \in X$. Then, there exist an additive function $A: X \rightarrow Y$, a quadratic function $B: X \rightarrow Y$, a cubic function $C: X \rightarrow Y$, and a quartic function $Q: X \rightarrow Y$ such that

$$
\begin{aligned}
\| f(x)-A(x)- & B(x)-C(x)-Q(x) \| \\
\leq & \frac{1}{|96| \mid k^{4}-} k^{2} \mid(1-L) \\
\times \max \{ & \left|2 k^{2}\right| \varphi(2 x, x),|2| \varphi(x, 2 x),|2| \varphi((1+k) x, x),|2| \varphi((1-k) x, x), \\
& \left|k^{2}\right| \varphi(2 x, 2 x), \varphi(x, x), \varphi(x, 3 x), \varphi((1+2 k) x, x), \varphi((1-2 k) x, x), \\
& \left|2 k^{2}\right| \varphi(-2 x,-x),|2| \varphi(-x,-2 x),|2| \varphi(-(1+k) x,-x), \\
& |2| \varphi(-(1-k) x,-x),\left|k^{2}\right| \varphi(-2 x,-2 x), \varphi(-x,-x), \varphi(-x,-3 x), \\
& \varphi(-(1+2 k) x,-x), \varphi(-(1-2 k) x,-x),\left|3\left(1-k^{2}\right)\right| \varphi(0, x), \\
& \frac{|3|}{|2|} \varphi(0,2 x),|3| \varphi(k x, x),\left|3\left(1-k^{2}\right)\right| \varphi(0,-x), \\
& \left.\frac{|3|}{|2|} \varphi(0,-2 x),|3| \varphi(-k x,-x)\right\}
\end{aligned}
$$

for all $x \in X$.

\section{Acknowledgments}

The authors would like to thank the area editor, Prof. Xue Zhong He, and two anonymous referees for their valuable comments and suggestions.

T. Z. Xu was supported by the National Natural Science Foundation of China (10671013).

\section{References}

[1] S. M. Ulam, A Collection of Mathematical Problems, vol. 8 of Interscience Tracts in Pure and Applied Mathematics, Interscience Publishers, London, UK, 1960.

[2] D. H. Hyers, "On the stability of the linear functional equation," Proceedings of the National Academy of Sciences of the United States of America, vol. 27, pp. 222-224, 1941.

[3] T. Aoki, "On the stability of the linear transformation in Banach spaces," Journal of the Mathematical Society of Japan, vol. 2, pp. 64-66, 1950.

[4] Th. M. Rassias, "On the stability of the linear mapping in Banach spaces," Proceedings of the American Mathematical Society, vol. 72, no. 2, pp. 297-300, 1978. 
[5] P. Găvruţa, "A generalization of the Hyers-Ulam-Rassias stability of approximately additive mappings," Journal of Mathematical Analysis and Applications, vol. 184, no. 3, pp. 431-436, 1994.

[6] J. M. Rassias, "Solution of a problem of Ulam," Journal of Approximation Theory, vol. 57, no. 3, pp. 268-273, 1989.

[7] J. M. Rassias, "Solution of the Ulam stability problem for quartic mappings," Glasnik Matematički. Serija III, vol. 34, no. 2, pp. 243-252, 1999.

[8] J. M. Rassias, "On approximation of approximately linear mappings by linear mappings," Journal of Functional Analysis, vol. 46, no. 1, pp. 126-130, 1982.

[9] K. Ravi, M. Arunkumar, and J. M. Rassias, "Ulam stability for the orthogonally general EulerLagrange type functional equation," International Journal of Mathematics and Statistics, vol. 3, no. A08, pp. 36-46, 2008.

[10] K. Ravi, J. M. Rassias, M. Arunkumar, and R. Kodandan, "Stability of a generalized mixed type additive, quadratic, cubic and quartic functional equation," Journal of Inequalities in Pure and Applied Mathematics, vol. 10, no. 4, article 114, pp. 1-29, 2009.

[11] T. Z. Xu, J. M. Rassias, and W. X. Xu, "Generalized Hyers-Ulam stability of a general mixed additivecubic functional equation in quasi-Banach spaces," Preprint.

[12] T. Z. Xu, J. M. Rassias, and W. X. Xu, "A fixed point approach to the stability of a general mixed additive-cubic functional equation in quasi fuzzy normed spaces," International Journal of Physical Sciences. to appear.

[13] T. Z. Xu, J. M. Rassias, and W. X. Xu, “Intuitionistic fuzzy stability of a general mixed additive-cubic equation," Journal of Mathematical Physics, vol. 51, no. 6, 21 pages, 2010.

[14] J. M. Rassias, "Solution of the Ulam stability problem for cubic mappings," Glasnik Matematički. Serija III, vol. 36, no. 1, pp. 63-72, 2001.

[15] Y. J. Cho, C. Park, and R. Saadati, "Functional inequalities in non-Archimedean Banach spaces," Applied Mathematics Letters, vol. 23, no. 10, pp. 1238-1242, 2010.

[16] M. E. Gordji and M. B. Savadkouhi, "Stability of a mixed type cubic-quartic functional equation in non-Archimedean spaces," Applied Mathematics Letters, vol. 23, no. 10, pp. 1198-1202, 2010.

[17] M. E. Gordji, H. Khodaei, and Th. M. Rassias, "On the Hyers-Ulam-Rassias stability of a generalized mixed type of quartic, cubic, quadratic and additive functional equations in quasi-Banach spaces," http:/ /arxiv4.library.cornell.edu/abs/0903.0834v2.

[18] A. Khrennikov, Non-Archimedean Analysis: Quantum Paradoxes, Dynamical Systems and Biological Models, vol. 427 of Mathematics and Its Applications, Kluwer Academic, Dordrecht, The Netherlands, 1997.

[19] D. Miheț and V. Radu, "On the stability of the additive Cauchy functional equation in random normed spaces," Journal of Mathematical Analysis and Applications, vol. 343, no. 1, pp. 567-572, 2008.

[20] A. K. Mirmostafaee, "Approximately additive mappings in non-Archimedean normed spaces," Bulletin of the Korean Mathematical Society, vol. 46, no. 2, pp. 387-400, 2009.

[21] M. S. Moslehian and T. M. Rassias, "Stability of functional equations in non-Archimedean spaces," Applicable Analysis and Discrete Mathematics, vol. 1, no. 2, pp. 325-334, 2007.

[22] M. S. Moslehian and G. Sadeghi, "A Mazur-Ulam theorem in non-Archimedean normed spaces," Nonlinear Analysis: Theory, Methods \& Applications, vol. 69, no. 10, pp. 3405-3408, 2008.

[23] M. S. Moslehian and G. Sadeghi, "Stability of two types of cubic functional equations in nonArchimedean spaces," Real Analysis Exchange, vol. 33, no. 2, pp. 375-383, 2008.

[24] A. Najati and G. Z. Eskandani, "Stability of a mixed additive and cubic functional equation in quasiBanach spaces," Journal of Mathematical Analysis and Applications, vol. 342, no. 2, pp. 1318-1331, 2008.

[25] B. Paneah, "Some remarks on stability and solvability of linear functional equations," Banach Journal of Mathematical Analysis, vol. 1, no. 1, pp. 56-65, 2007.

[26] C. Park, "Fixed points and the stability of an AQCQ-functional equation in non-archimedean normed spaces," Abstract and Applied Analysis, vol. 2010, Article ID 849543, 15 pages, 2010.

[27] R. Saadati, S. M. Vaezpour, and Y. J. Cho, "A note to paper "On the stability of cubic mappings and quartic mappings in random normed spaces", " Journal of Inequalities and Applications, vol. 2009, Article ID 214530, 6 pages, 2009.

[28] T. Z. Xu, J. M. Rassias, and W. X. Xu, "A generalized mixed quadratic-quartic functional equation," Bulletin of the Malaysian Mathematical Sciences Society. to appear.

[29] A. M. Robert, A Course in p-Adic Analysis, vol. 198 of Graduate Texts in Mathematics, Springer, New York, NY, USA, 2000.

[30] V. S. Vladimirov, I. V. Volovich, and E. I. Zelenov, p-Adic Analysis and Mathematical Physics, vol. 1 of Series on Soviet and East European Mathematics, World Scientific, River Edge, NJ, USA, 1994. 
[31] G. Isac and Th. M. Rassias, "Stability of $\psi$-additive mappings: applications to nonlinear analysis," International Journal of Mathematics and Mathematical Sciences, vol. 19, no. 2, pp. 219-228, 1996.

[32] V. Radu, "The fixed point alternative and the stability of functional equations," Fixed Point Theory, vol. 4, no. 1, pp. 91-96, 2003.

[33] J. B. Diaz and B. Margolis, "A fixed point theorem of the alternative, for contractions on a generalized complete metric space," Bulletin of the American Mathematical Society, vol. 74, pp. 305-309, 1968. 


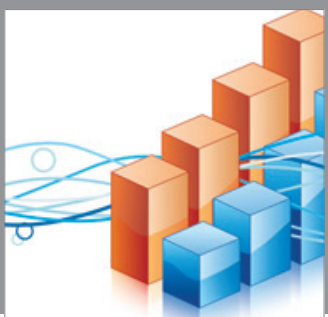

Advances in

Operations Research





\section{The Scientific} World Journal
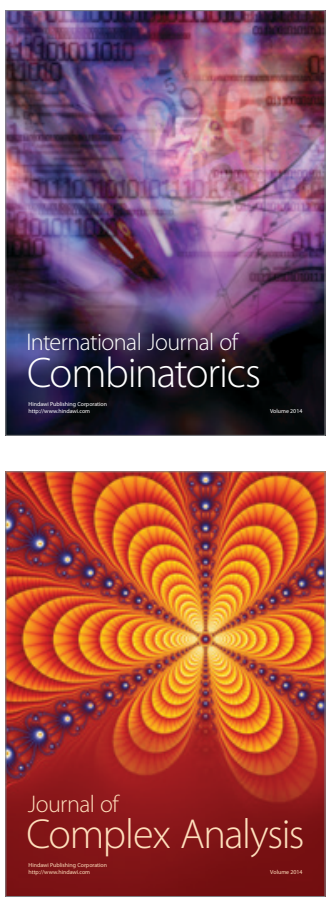

International Journal of

Mathematics and

Mathematical

Sciences
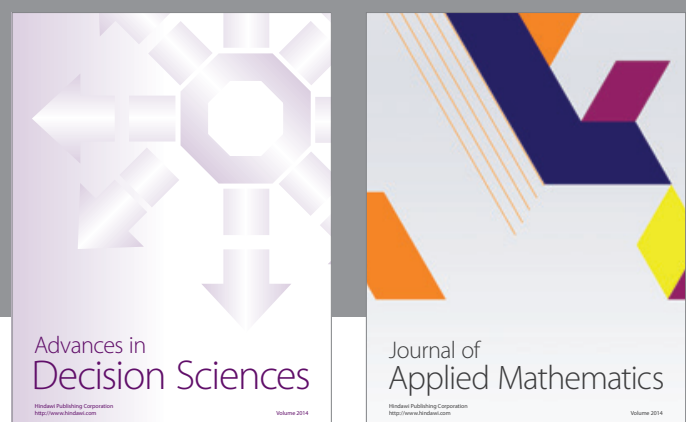

Journal of

Applied Mathematics


Submit your manuscripts at http://www.hindawi.com


Mathematical Problems in Engineering


Journal of

Function Spaces


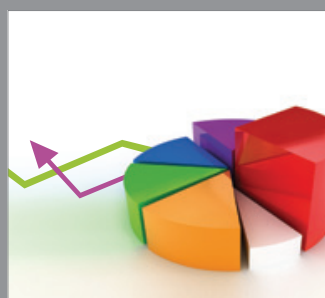

ournal of

Probability and Statistics

Promensencen
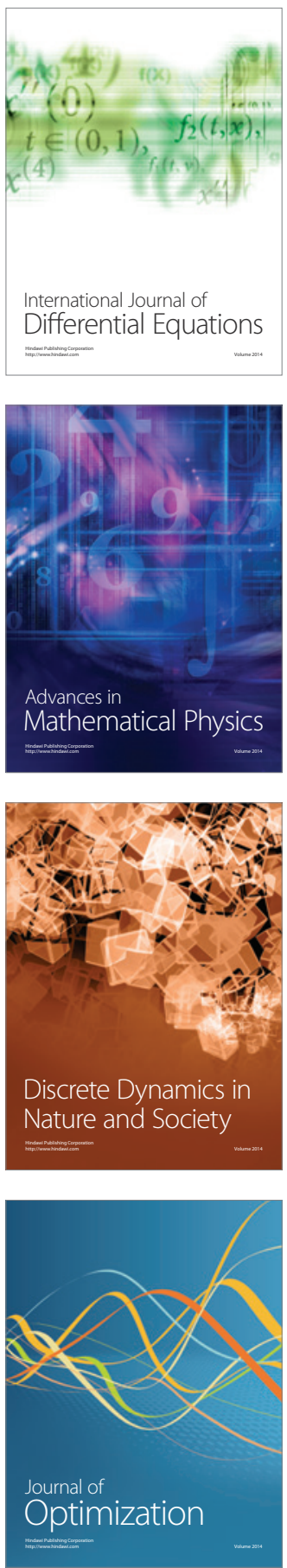